\title{
eJRIEPS
}

Ejournal de la recherche sur l'intervention en éducation physique et sport

49 | 2021

Varia

\section{Modèle d'apprentissage des décisions tactiques : une approche centrée sur les élèves}

Tactical-decision learning model: a student-centered approach

Jean-Francis Gréhaigne, Nathalie Wallian, Paul Godbout et Marie-Paule Poggi

\section{OpenEdition}

\section{Journals}

Édition électronique

URL : http://journals.openedition.org/ejrieps/5999

DOI : 10.4000/ejrieps.5999

ISSN : 2105-0821

Éditeur

ELLIADD

\section{Référence électronique}

Jean-Francis Gréhaigne, Nathalie Wallian, Paul Godbout et Marie-Paule Poggi, « Modèle

d'apprentissage des décisions tactiques : une approche centrée sur les élèves », eJRIEPS [En ligne],

49 | 2021, mis en ligne le 01 avril 2021, consulté le 08 avril 2021. URL : http://journals.openedition.org/ ejrieps/5999; DOI : https://doi.org/10.4000/ejrieps.5999

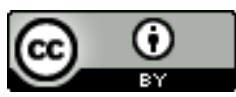

La revue eJRIEPS est mise à disposition selon les termes de la Creative Commons Attribution 4.0 International License. 


\section{Modèle d'apprentissage des décisions tactiques:}

une approche centrée sur les élèves

Jean-Francis Gréhaigne ${ }^{\star}$, Nathalie Wallian ${ }^{* *}$, Paul Godbout ${ }^{* \star *} \&$ Marie-Paule Poggi ${ }^{* * *}$

* Université de Bourgogne Franche-Comté

** Université de la Réunion

*** Université Laval, Québec, Canada

**** Université des Antilles

Résumé

En éducation physique et sportive, on trouve encore une utilisation de séances d'entraînement type club complètement inadaptées au temps effectif dont on dispose à l'école. Ce constat nous conduit à proposer une autre approche de l'apprentissage des sports collectifs. L'utilisation de l'approche tactique comme cadre de référence, ainsi que la contribution d'une perspective constructiviste et cognitiviste en relation avec les travaux sur les connaissances et compétences motrices dans les sports d'équipe nous ont amenés, dans le cadre de l'éducation physique à l'école, à mettre en avant le "modèle d'apprentissage des décisions tactiques ". Ce modèle est basé sur l'exploration, par les élèves, des diverses possibilités dans des jeux réduits, en vue de la construction de réponses nouvelles.

Mots clés : sports collectifs, apprentissage, conceptualisation, débat d'idées, pratique réflexive.

Tactical-decision learning model: a student-centered approach

Summary

In physical education, there is still a use of club-type coaching sessions which are completely unsuitable for the effective time available at school. This observation leads us to propose another approach to team-sport learning. The use of the tactical approach as a frame of reference, as well as the contribution of a constructivist and cognitivist perspective in relation to the work on knowledge and motor skills in team sports led us, within the framework of the physical education at school, to highlight the "tactical-decision learning model". This model is based on the exploration, by the students, of the various possibilities in small-sided games, in view of building new responses.

Keywords: team sports, learning, conceptualization, debate of ideas, reflective practice. 


\section{Introduction}

À l'heure actuelle, l'intérêt pour l'enseignement / apprentissage des sports d'équipe est illustré par une augmentation notable des écrits sur les conceptions des jeux et les différentes approches de leur enseignement (Harvey \& Jarrett, 2014). Néanmoins, 55 ans d'innovation et de propositions (du Colloque Vichy de 1965 aux programmes de 2020) n'ont pas suffi à vraiment rénover les contenus d'enseignement dans les leçons de sport collectif. Le modèle d'apprentissage des décisions tactiques (MADT) que nous allons détailler vise, en partie, à remédier à cet état de fait.

Les leçons de sport collectif préparées par nos étudiants en éducation physique et qui, de ce point de vue, nous semblent rendre compte de la représentation dominante de la « bonne leçon ", sont construites presque toujours sous la même forme. Une première partie est consacrée à un échauffement avec ou sans ballon. Une deuxième partie, corps principal de la leçon, va de l'étude des gestes spécifiques de l'activité considérée à des situations simplifiées avec ou sans opposition. Enfin, en fonction du temps disponible, dans une troisième partie, le jeu ou les situations jouées sont utilisés (Gréhaigne, 1992). En éducation physique et sportive (EPS), ce type d'approche se révèle, souvent, complètement inadapté au temps effectif disponible à l'école. Ce constat nous conduit, une nouvelle fois, à proposer une réflexion sur l'enseignement-apprentissage des sports collectifs.

\subsection{Mouvance initiale}

La première remise en cause de l'omnipotence du modèle technique et individualiste qui a longtemps été à la base de l'enseignement des jeux et des jeux sportifs collectifs (JSC) date du début des années 60 sous l'influence de Fréderich Mahlo (1969) avec l'étude de l'acte tactique en jeu et a amorcé les prémices de la recherche sur le jeu collectif. En France, le colloque de Vichy sur l'enseignement des jeux sportifs collectifs (JSC) (1964-65) est organisé autour de trois thèmes : les principes communs à tous les Jeux Sportifs ; la formation du joueur depuis l'initiation jusqu'à la compétition ; la place du maitre dans l'apprentissage de telle ou telle spécialité (Amicale des Anciens Élèves de l'ENSEP, 1966). Une liaison théorie / pratique est proposée avec une organisation spécifique de la classe (équipes premières et réserves) structurée en club $A$ et $B$ avec un calendrier (progression en vue de compétitions). Du stage d'expérimentation comparée de Malakoff en 1964 jusqu'à la fin des stages Maurice Baquet de Sète en 1980, la Fédération Sportive et Gymnique du 
Travail (FSGT) a initié et promu l'innovation et la recherche-action dans les activités physiques avec, comme point incontournable, partir de ce que sait faire l'enfant.

\subsection{Des précurseurs en France}

En 1974, commence la publication par le Conseil Pédagogique et Scientifique (CPS) de la Fédération de 12 mémentos, dont 10 pour chaque activité physique pratiquée dans la colonie «Gai soleil » à Sète. Chacun des mémentos comporte trois parties: (a) des situations pédagogiques sous forme de bandes dessinées ; (b) une observation des gestes typiques de l'évolution du débutant vers la conquête d'une motricité adaptée ; et (c) un essai de compréhension du haut niveau, pour caractériser une succession de niveaux d'adaptation. Le contenu de ces ouvrages est particulièrement représentatif des options retenues à cette époque pour analyser l'activité des enfants confrontés, par exemple, à la pratique des sports collectifs (cf. Alin, Bourrier, Buono, Jade, \& Martinez, 1979, pour un exemple en football). Il est toutefois à noter dès à présent que le choix est fait par Robert Mérand et le CPS de se placer délibérément dans une démarche expérimentale en pédagogie. Ce processus méthodologique vise la distanciation et comprend deux temps : un temps d'observation destiné à étudier, en différé (avec l'aide de la vidéo), ce qui s'est effectivement passé et un temps d'analyse, d'interprétation et d'incorporation de connaissances (Mérand, 1977). La démarche part donc du réel de la pratique et propose une interprétation des traces d'activité (la vidéo étant tout récemment disponible pour le commun des enseignants) visant à extraire des connaissances de la pratique.

Deleplace (1966 ; 1979), au-delà du rugby, apporte, dans une perspective différente, une contribution conséquente à la modélisation, l'enseignement et l'entraînement des sports collectifs. II fournit ainsi une méthodologie didactique, mais aussi une démarche pédagogique et une nomenclature d'exercices où apprendre le jeu dans le jeu s'opère en opposition réelle, modulée, raisonnée et tient une place centrale. La notion de matrice d'action est présentée et conçue comme une enveloppe générale qui peut donner lieu à une complexification interne croissante (vers le particulier et le complexe). La prise de conscience des propriétés du jeu permet au joueur d'orienter celui-ci à partir d'un schéma abstrait qui rend compte, de façon opératoire au niveau mental, de la logique d'action correspondant à la situation évolutive particulière à laquelle se rapporte la configuration du jeu en cours. Ce processus se réalise de façon opératoire, c'est-à-dire d'une façon qui permet au joueur d'agencer progressivement les éléments du jeu en fonction des cas de figure rencontrés. Le joueur réussit grâce à une faculté d'anticipation mentale dans 
l'élaboration des suites de décisions au fil de l'action et sous temps contraint. Enfin, Deleplace (1979) approfondit le concept d'unité dialectique des rapports d'opposition attaque $<=>$ défense en rapport avec le référentiel commun de la logique interne du jeu.

\subsection{Vers une pédagogie des modèles de décision tactique}

Dans sa thèse, Bouthier (1988) a étudié et comparé différentes options concernant l'enseignement des sports collectifs en distinguant la pédagogie des modèles d'exécution (PME) de la pédagogie des modèles auto-adaptatifs (PMAA) et de la pédagogie des modèles de décision tactique (PMDT). Cette dernière a obtenu expérimentalement de meilleurs résultats que les deux premières. Néanmoins, une approche centrée sur les objets de la technique (Gréhaigne, 2016) reste très utilisée en EPS. Bouthier (1988) qualifie alors cette forme sociale de transmission des savoirs spécifiques aux activités physiques et sportives de «pédagogie des modèles d'exécution» (PME). Celle-ci postule que les apprentissages passent par la reproduction des modèles techniques et la maitrise par les joueurs des solutions les plus efficaces. Cette conception « applicationniste » de la technique postule une équivalence entre la situation d'entraînement et les situations de jeu. Cette pédagogie des modèles d'exécution devrait être remplacée par « la pédagogie des modèles de décision tactique » (PMDT) qui postule que l'intervention des processus cognitifs est décisive dans l'orientation et le contrôle moteur des actions. Elle engage la construction, par le sujet, de repères perceptifs significatifs et de principes rationnels qui facilitent les choix tactiques sous contrainte temporelle. Ces repères influent sur la qualité de l'exécution en termes de timing et de synchronisation des actions dans le temps. Ici « comment le faire et quand le faire » deviennent un aspect fondamental de la gestion des rapports d'opposition.

Plus diffusé dans le système scolaire, le «modèle d'apprentissage des décisions tactiques », sur lequel nous discuterons plus avant, entretient une filiation forte avec la PMDT. En poursuivant dans la même direction, Darnis, Lafont et Menaut (2007) ont montré l'intérêt des interactions langagières en dyades plutôt dissymétriques pour un apprentissage des choix tactiques au handball avec des jeunes filles de classes de 6ème. Le recours au langage, en tant qu'outil ou instrument de la pensée (Vygotski, 1985 [1934]), favorise une prise de conscience et l'émergence des règles de l'action efficace ainsi que de la pensée tactique. 


\section{Un modèle pour l'enseignement / apprentissage à l'école}

L'acte d'apprendre constitue une action d'auto-transformation qui vise une construction de nouvelles connaissances et de compétences. Un modèle pose un certain nombre de questions et ne répond qu'à certains types de problème. Une modélisation du système d'enseignement / apprentissage à l'école est fondée, de façon explicite ou non, sur des conceptions (Walliser, 1977).

\subsection{Les composantes du modèle}

Cette modélisation est basée sur l'exploration, par les élèves, des diverses possibilités dans des jeux réduits, en vue de la construction de réponses nouvelles. Effectivement, l'utilisation de l'approche tactique comme cadre de référence, ainsi que la contribution d'une perspective constructiviste et cognitiviste (Kozanitis, 2005) en relation avec les travaux sur les connaissances et compétences motrices dans les sports d'équipe (Ennis, 2007 ; Gréhaigne \& Godbout, 1995 ; Gréhaigne, Godbout, \& Bouthier, 1999 ; Gréhaigne, Godbout, \& Bouthier, 2001) nous ont amenés, dans le cadre de l'éducation physique à l'école, à mettre en avant le « modèle d'apprentissage des décisions tactiques » (MADT) .

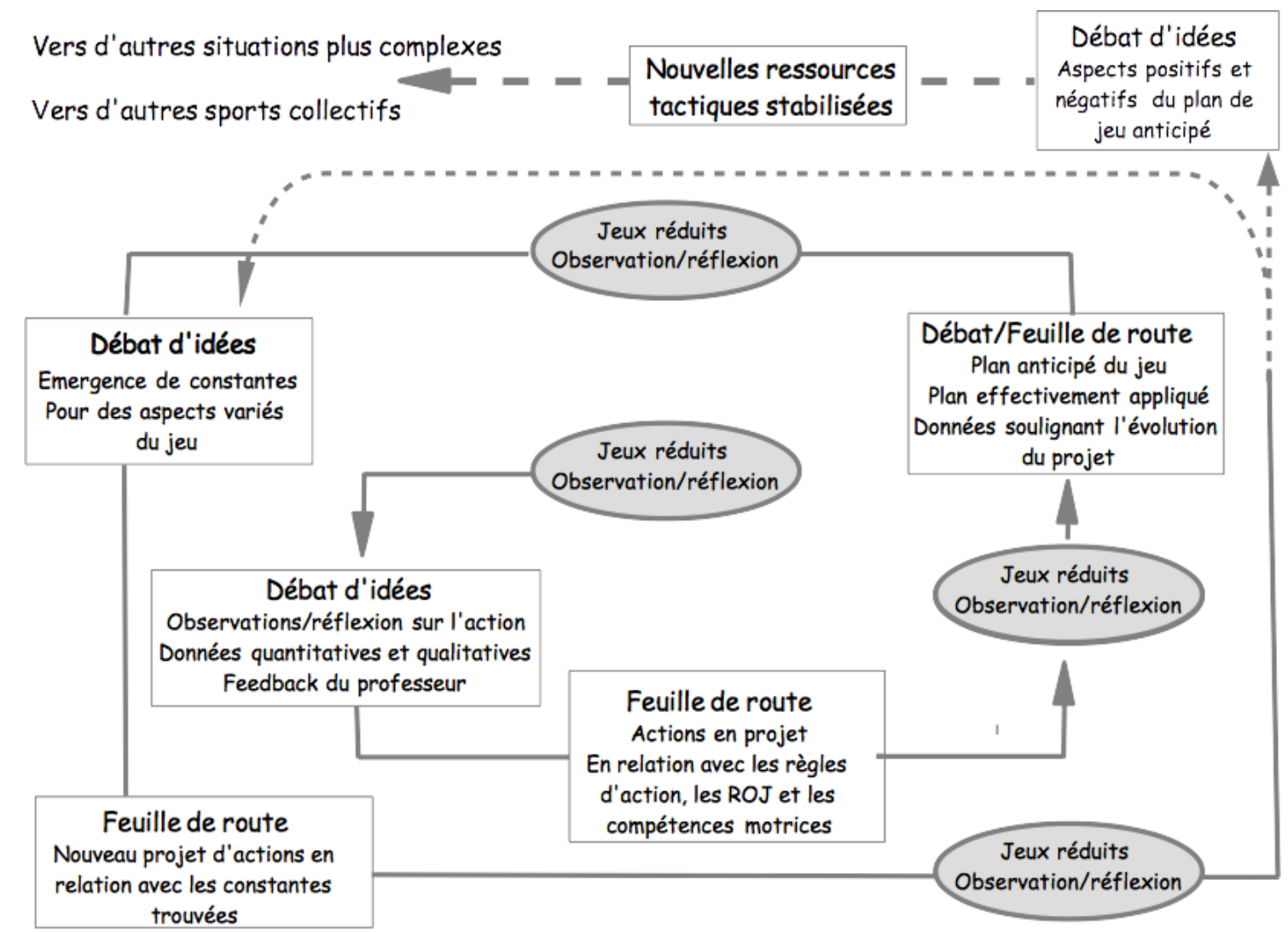

Figure 1. Modèle d'apprentissage des décisions tactiques (traduit et adapté de Gréhaigne, Wallian et Godbout, 2005). 
Le MADT est basé sur l'exploration réflexive, négociée par les élèves, des diverses alternatives décisionnelles dans des jeux à effectifs réduits (JER) en vue de la construction de réponses inédites.

La figure 1, originalement inspirée des travaux de Gréhaigne et Godbout (1998b), de Gréhaigne, Billard et Laroche (1999) et de Gréhaigne, Wallian et Godbout, (2005), illustre notre modèle d'enseignement opérationnel qui devrait améliorer la construction des connaissances tactiques par les élèves et le développement de leurs compétences en matière de prise de décision. Dès le début de la séquence d'apprentissage, les élèves sont mis en action dans un JER ( $3 \times 3$ ou $4 \times 4$ par exemple). La volonté d'associer les élèves à la construction de leurs connaissances et compétences motrices est claire (Gréhaigne, 1995 ; Gréhaigne et al., 2005).

Cette modélisation propose cinq composantes différentes qui sont utiles aussi bien dans les « situations de référence», les «situations d'apprentissage » que les «situations d'évaluation » (Gréhaigne, Billard, Guillon, \& Roche, 1989).

* La composante « action » où les élèves sont en activité motrice dans un match (jeu réduit). Cette situation de jeu constitue, dans un ensemble finalisé par un objectif de production (le gain du match), la rencontre de deux équipes dans une situation fondamentalement réversible. C'est la situation classique où des élèves, confrontés à une tâche proposée, le plus souvent, par l'enseignant, jouent et essaient de résoudre le ou les problèmes posés par la confrontation.

* La composante « observation » où les élèves qui ne participent pas aux activités motrices relèvent des informations en fonction de critères chiffrés ou qualitatifs définis précisément. II peut être organisé une observation participante pour la régulation du jeu en cas de feuille de route. Ces données collectées serviront pour l'évaluation du jeu et les prises de décision lors du débat à venir. En effet, passer du temps à observer ou à relever des données est aussi, pour les élèves, une situation d'apprentissage effective où ils apprennent à décoder des comportements et à reconnaître les configurations du jeu (Chang, 2009 ; Godbout \& Desrosiers, 2005 ; Ramos, Coutinho, Davids, \& Mesquita, 2020).

* La composante « débat d'idées » (Deriaz, Poussin, \& Gréhaigne, 1998 ; Gréhaigne \& Godbout, 1998b) : ce sont des situations dans lesquelles les élèves s'expriment et échangent à propos du jeu. Le débat d’idées consiste, après une séquence jouée, en une discussion destinée à faire évoluer ou non le projet d'action de l'équipe. Il s'agit, en fonction des observations recueillies, de reconsidérer ou non la stratégie prévue et la tactique effectivement appliquée. 
* La composante « feuille de route » permet aux élèves de consigner des hypothèses d'action, une stratégie à venir. En général, cette expression désigne le recensement des moyens, des règles et des priorités à adopter en fonction des tâches proposées pour atteindre les objectifs fixés. Nous y reviendrons plus avant.

* La composante « itérations » offre la possibilité aux élèves de répéter à plusieurs reprises le cycle hypothèse/vérification dans l'action, conclusion et régulation. En effet, en éducation, une itération est définie comme un processus d'apprentissage et de développement où l'on incite les élèves à répéter des expériences, des évaluations ou des projets, jusqu'à ce que des progrès soient constatés.

\subsection{Apprentissage au travers de la compréhension}

Dans ces circonstances, l'apprentissage des jeux au travers de la compréhension (AJtC) (Chandler, 1996 ; Gréhaigne, Caty, \& Godbout, 2010) privilégie l'utilisation judicieuse du jeu car cela permet de conserver le côté ludique indispensable à toute bonne séance d'éducation physique. Le but sous-jacent est bien d'aider les élèves à découvrir les problèmes puis à les résoudre dans des conditions adaptées à leur niveau. II ne s'agit donc pas de proposer des solutions miracles mais d'offrir des pistes de réponses possibles pour avancer sur comment « jouer le jeu ». Enseigner au travers de la compréhension vise à réduire les apports de produits prêts à l'emploi, conçus et élaborés ailleurs et destinés à des enseignants simples applicateurs. Cela nécessite une rupture qui repose sur le passage d'une conception normative du prêt-à-agir didactique à une approche réflexive qui permet de conceptualiser et d'apprendre en situation de jeu (Ramos et al., 2020).

Les progrès chez les joueurs et les joueuses impliquent non seulement l'apparition de réponses nouvelles face à un problème donné, mais aussi une stabilisation de ces réponses (Poggi, 2016). Tout ce processus nécessite une réflexion de la part des élèves pendant une période qui peut être très variable selon les capacités intellectuelles des élèves et leur vécu sportif. La prise de conscience d'un fait, d'un fonctionnement, d'une émotion est un processus qui fait passer ces éléments d'une activité non-consciente, voire automatique, à une activité consciente qui sait expliciter et mettre en mots des faits, des ressentis, des initiatives, des tactiques collectives (Deleplace, 1979 ; Gréhaigne, Godbout, \& Mahut, 1999). Il faut que les élèves soient conscients des stratégies d'apprentissage qu'ils mettent en œuvre pour apprendre et comprendre le jeu. Cette conceptualisation suppose une capacité de prise de distance, de l'abstraction et le déploiement d'une activité langagière 
(verbale et non verbale) qui renvoie au développement ou à la construction de connaissances nouvelles à partir de l'expérience.

Cette approche didactique est fondée sur l'idée d'une joueuse ou d'un joueur actif qui construit de nouvelles compétences ou connaissances reposant sur ou inhibant celles déjà en place. Les enseignants ont trop longtemps considéré qu'il suffisait d'enseigner pour que les élèves apprennent : or la manière d'enseigner est désormais indissociable de la façon d'interpréter le fonctionnement de l'élève. En effet, son mode de construction des connaissances et des compétences motrices devient premier (Gréhaigne, Poggi, \& Zerai, 2017). Tout dispositif didactique classique suppose la mise en place d'un contrat didactique particulier où l'apprenant, pour exercer son métier d'élève, est appelé à remplir des tâches différenciées selon des niveaux de difficulté indexés et échelonnés. II s'agit classiquement d'un métier d'élève dans lequel la situation d'apprentissage imposée sous forme d'exercices se présente selon des modalités définies par d'autres et faisant l'objet d'un contrôle permanent aux fins d'obtenir le monitoring contrôlé de la personne (Perrenoud, 1994).

Avec les pédagogies constructivistes, faites de questionnements, d'interlocutions, d'initiatives, on touche non seulement à l'implication active dans une tâche ouverte, mais aussi aux conditions mêmes de la réussite (Wallian \& Chang, 2007).

\section{Les jeux à effectifs réduits}

En éducation, le jeu peut être un formidable instrument d'apprentissage, de promotion de la mixité et de développement de l'esprit d'équipe (Gréhaigne, 2019).

\subsection{Le jeu : un contexte de réflexion et de motivation}

L'approche des JERs permet au débutant d'enrichir son niveau de compétence actuel et son expertise se développe en termes de perception du jeu, de sens tactique, de prise de décisions et d'exécution (Kirk \& McPhail, 2002). Fait important, les JERs impliquent le développement de compétences telles que la réflexion stratégique et la résolution de problèmes, deux aspects importants mais souvent sous-estimés car renvoyant aux habiletés cognitives de haut niveau (Resnick, 1987). Le choix d'un jeu à effectif réduit (4x 4 par exemple) limite le nombre d'informations à prélever pour le joueur novice. Dans une équipe comprenant un nombre de joueurs plus important, les informations essentielles sont noyées dans un ensemble trop vaste, et la perception est le plus souvent asservie à l'émotion. En jouant avec trois ou quatre partenaires, il est possible, en tous cas plus facile, de percevoir les différentes alternatives fondamentales (Kawa, 1998). Les élèves sont aussi 
davantage impliqués dans les jeux (plus de mouvements et de situations) car il y a moins de joueurs sur le terrain. Les plus faibles sont sûrs de toucher la balle plus souvent ce qui garantit une plus grande attention individuelle de chacun et des progrès. Les joueurs ont aussi plus d'opportunités de marquer des buts. Le ballon change souvent d'équipe car même si les séquences de jeu deviennent un peu plus longues, elles dépassent rarement quatre ou cinq échanges de balle. Les élèves sont beaucoup plus solidaires car ils sont à la fois impliqués dans les actions d'attaque et de défense, étant ainsi plus souvent exposés à toutes sortes de situations de jeu. Dans les jeux réduits, chaque joueur ou joueuse joue tout le temps, reçoit le ballon souvent, essaie de marquer en permanence, possède une plus grande liberté de jouer et, de ce fait, prend plus de plaisir.

\subsection{Action située mais adaptée}

Pourquoi ce choix des jeux réduits ? Les jeux réduits sont un excellent moyen de mettre les joueurs dans des situations de jeu et d'aider à leur développement. Ces jeux sont un moyen populaire d'entraîner des joueurs de tous âges, du débutant au niveau professionnel. Le format réduit propose aux joueurs plusieurs scénarios de type jeu dans un espace qui doit être compatible avec leurs ressources. On peut également y manipuler les règles, des restrictions pour mettre l'accent sur les différents aspects tactiques du jeu qui vont entraîner des progrès techniques. Quand on utilise des jeux réduits dans l'apprentissage, les joueurs jouent à une version modifiée de la pratique sociale de référence qui devrait leur permettre de construire des connaissances et des compétences motrices en situation. Le jeu réduit conserve les éléments importants de la complexité tactique, mais permet une exécution avec un niveau d'exigences physiques (espace réduit) et un temps plus important (moindre densité des joueurs) qui correspondent mieux au niveau des débutants (Nadeau, Gréhaigne, \& Godbout, 2017 ; Zerai, Gréhaigne, \& Godbout, 2011). Au plan de la sémiotique, dans ces jeux à effectif réduit, les signes contenus dans le jeu deviennent une sorte de « texte » qui peut être constamment lu et interprété à qui sait les lire.

Plusieurs éléments relevant de l'expérience ou de recherches complètent ces données sur le bénéfice que l'on peut retirer des jeux réduits en comparaison avec le football à 11. En général, les joueurs touchent le ballon cinq fois plus souvent au football à $4 \times 4$ et $50 \%$ plus qu'au football à $6 \times 6$ (Gréhaigne, \& Caty, 2014). Ils sont trois fois plus souvent confrontés au duel pour la conquête ou la conservation du ballon en football à 4 et deux fois plus souvent qu'au football à $6 \times 6$. Les buts sont marqués en moyenne toutes les minutes au football à $4 \times 4$ et toutes les quatre minutes au football à $6 \times 6$. La dimension des terrains est elle aussi 
très importante. Elle doit être adaptée à l'âge, aux compétences tactiques et motrices en relation avec le nombre de joueurs. Une surface comprise entre 60 et $100 \mathrm{~m}^{2}$ par joueur semble un bon compromis.

La durée des situations constitue aussi une variable essentielle : pas trop longue afin d'éviter une fatigue trop importante qui a pour conséquence une baisse évidente de l'efficience des élèves ; pas trop courte afin de laisser le temps aux élèves de s'adapter aux conditions de jeu proposées. Une durée de 6 à 8 minutes paraît réaliste pour éviter ces deux écueils (Marle \& Gréhaigne, 2009). Cette durée permet également d'effectuer un grand nombre de rotations entre les équipes et évite ainsi les temps morts, sources de comportements déviants. Enfin, la taille des cibles représente des éléments incontournables des sports collectifs. En effet, ces dernières sont porteuses de sens pour les joueurs et orientent leurs initiatives en fonction de la réussite. II semble donc indispensable, du moins dans un premier temps, de proposer des cibles larges et / ou en grand nombre. Ensuite, quand le jeu devient plus efficient (et que l'on ne souhaite pas immobiliser un joueur dans les buts), la taille de la cible peut être réduite avec l'utilisation, par exemple, d'une haie retournée. En effet, une cible avec une faible surface favorise davantage la construction du jeu pour aller au but que la recherche du tir. II peut s'agir d'une alternative intéressante sur le plan scolaire car elle évite la domination des élèves meilleurs tireurs au détriment des autres. Enfin, l'utilisation des jeux réduits facilite, par sa clarté, l'apprentissage des règles du jeu et de rapports d'opposition respectueux de l'adversaire.

II s'agit donc de proposer des activités authentiques plutôt que des exercices sans ballon ou avec ballon sans adversaires. Les jeux à effectif réduit proposent des situations de pratique adaptées où, d'emblée, les joueurs sont confrontés à ce qui est le plus important dans l'activité pratiquée : des adversaires et des partenaires. Ainsi, aussitôt, on cultive l'autonomie de chacun des participants et, en même temps, la réussite de tous par la proposition de situations de jeux dans lesquelles l'élève, même débutant, dispose déjà de quelques réponses. Enfin, ces situations de jeu réduit doivent permettre à tous de développer leurs propres réponses et d'en inventer de nouvelles pour progresser.

\subsection{Regroupement des élèves}

La façon dont on regroupe les élèves avec, en premier, des groupes mixtes ou pas, conditionne bien sûr la qualité des débats d'idées. Dans ce cas, il semble primordial de penser à mettre en commun le travail de chaque équipe pour éviter les laissés-pour-compte. Il est aussi nécessaire d'assurer une stabilité du groupe de manière à préserver une certaine 
continuité dans l'efficacité de l'équipe afin d'aider chacun de ses membres à progresser. Comme il n'y a pas de réponse toute faite aux problèmes posés par la constitution des équipes de sports collectifs en EPS, les modalités de groupement sont à envisager en fonction de la population effective de la classe. Néanmoins ces groupements renvoient, le plus souvent, à quelques grandes catégories.

- Groupes homogènes entre eux mais hétérogènes dans leur composition : tout le monde est mélangé, on préserve simplement l'égalité des chances entre toutes les équipes ;

- Groupes homogènes entre eux et homogènes dans leur composition. Ce sont les groupes de niveaux où les meilleurs sont regroupés, les plus faibles formant un autre groupe ;

- Groupes d'élèves de faible niveau avec un bon joueur pour mener le jeu. C'est une modalité qui est peu utilisée mais qui peut donner de bons résultats avec des élèves plus âgés ;

- Groupes affinitaires qui peuvent servir à démarrer une activité avec des classes mixtes. Cependant, ceux-ci sont à manier avec précaution car très vite les apprentissages des élèves stagnent au profit du plaisir d'être ensemble :

- Groupes de besoins qui sont formés avec des élèves présentant des types de difficultés identiques, mais leur niveau de performance pouvant être très varié. II est important que cette forme de groupement ne devienne pas une forme d'exclusion.

II s'agit donc d'utiliser la diversité comme source de progrès pour tous et toutes en oubliant parfois le modèle sportif dominant qui exclut et normalise tout en maintenant le pouvoir décisionnel dans les mains du formateur ou de l'entraîneur.

\subsection{Se donner du temps et gérer un changement de métier}

Néanmoins, quel que soit le mode de groupement choisi, le jeu, le débat d'idées et les actions en projet exigent du temps, du travail pour envisager des progrès. Élèves et enseignants ne sont pas forcément prêts à s'y engager car, dans ce cas, leurs rapports habituels sous la forme d'un contrat didactique classique (Brousseau, 1986) se trouvent modifiés ; cela touche au métier d'élève et au métier de professeur, ce qui explique en partie la difficulté à s'engager dans ce type de pratiques pédagogiques. Assurément, la nature du rôle et de l'activité de l'enseignant change : elle va consister plus à attirer l'attention de l'élève sur des façons possibles de faire, sur les moyens employés pour réussir et sur la spéculation entre le projet et les issues possibles et avérées. A partir d'indicateurs de la 
pratique, l'élève devra effectuer une mise en rapport attentive et critique entre le résultat de l'action et des indicateurs relatifs à l'activité, en relation avec les données objectives collectées. De la sorte, les élèves deviennent progressivement des acteurs de leurs apprentissages tout en étendant la marge d'initiative du métier d'élève (Gréhaigne, Mahut, \& Godbout, 2000).

L'attachement d'un élève ou d'un enseignant au « métier traditionnel » peut être une cause de forte résistance au changement et c'est bien la nature de la relation au savoir qui est en question.

\section{Observer et évaluer en sport collectif : des éléments quantitatifs et qualitatifs}

Quelques aspects de l'approche interactionniste socio-discursive nous paraissent particulièrement intéressants (Bronckart, 1996). Ainsi, lors de la production d'interlocutions à propos du jeu, le joueur doit utiliser des données sur le jeu présentes dans sa mémoire. Ces traces mnésiques comparées au présent de la tâche constituent des références qui seront à la source de toute décision éclairée par l'expérience passée. Voyons maintenant au plan pratique comment peut être mise en œuvre cette démarche réflexive et interprétative de l'action de l'élève.

\subsection{La nature des informations prélevées}

Dans les activités collectives telles que les sports collectifs, un modèle bidimensionnel a été proposé pour identifier le type d'information que pourrait rechercher un observateur (Gréhaigne, Godbout, \& Bouthier, 1997 ; Nadeau, Richard, \& Godbout, 2008). L'une de ces dimensions a trait à la distinction technique / tactique, à savoir si l'information recherchée relève du domaine des gestes moteurs ou du domaine des choix tactiques et stratégiques. L'autre dimension a trait à la distinction processus / produit, à savoir si l'observateur cible la façon dont l'élève accomplit le geste ou la tâche visée, ou le résultat final de son action. L'information captée par l'observateur peut donc être de type :

- technique-produit (un joueur vient d'effectuer une passe à Marie. Marie réussit-elle à prendre possession de la balle ?) ;

- technique-processus (Pierre effectue une réception de service en volley-ball. De quelle façon s'y prend-il pour exécuter ce geste ?) ;

- tactique-produit (Denis, en situation de jeu au hockey sur glace, est marqué par un adversaire. Arrive-t-il à se démarquer suffisamment pour devenir un receveur potentiel ? ; 
- tactique-processus (comment Denis s'y prend-il pour se démarquer de son adversaire direct ?).

Dans un contexte d'apprentissage socioconstructiviste tel que préconisé par le MADT, sachant que l'observateur communiquera ses résultats à ses co-équipiers, ce que ces derniers veulent connaître, ce sont des informations de type produit afin de construire des solutions appropriées en termes de processus ou de façons de faire. Ainsi, à titre d'exemple, des résultats tels que le nombre de balles conquises, le nombre de balles perdues, le nombre de tirs au but, etc., (voir Gréhaigne \& Godbout, 1998a ; Gréhaigne et al., 1997; Gréhaigne \& Marle, 2016) constituent des données à la fois de type technique-produit et de type tactique-produit. II revient aux élèves, lors du débat d'idées et de l'inscription d'un projet d'action dans la feuille de route, d'opter pour du travail d'apprentissage sur de la technique et/ou sur des aspects tactiques.

L'utilisation de données chiffrées peut consister à enregistrer des comportements jugés signifiants d'une adaptation à une modélisation du jeu (Brau-Antony, \& Cleuziou, 2005 ; Gréhaigne, 1989 ; Mérand, 1984) soit pour l'équipe, soit pour les joueurs. Pour l'équipe, reprenant l'exemple énoncé plus haut, le simple fait d'analyser les rapports entre le nombre de buts marqués, le nombre de tentatives de tirs et le nombre d'attaques (ce qui revient à compter le nombre d'entrées en possession du ballon pour l'équipe) dans des rencontres de durée constante, donne des indications très précieuses. En effet, l'efficacité est une donnée centrale en sport collectif car pour gagner il faut marquer un point de plus que l'adversaire. Les comportements des joueurs s'inscrivent et s'insèrent dans des configurations du jeu temporaires illustrant un rapport d'opposition momentané.

Pour obtenir des informations encore plus précises sur les conditions de l'affrontement, on a également utilisé différents indices chiffrés. Nous définirons par «indice » une valeur numérique obtenue en mettant en rapport la fréquence d'apparition de deux comportements. Celui-ci ne doit pas être considéré comme une mesure absolument exacte, car le choix des éléments retenus comporte toujours une part d'arbitraire. Néanmoins, utilisés avec prudence, les indices constituent souvent le seul moyen d'avoir une connaissance approchée sur des phénomènes complexes qui président au jeu et sur leurs conséquences en matière décisionnelle (Gréhaigne, 2019). Par exemple, «l'indice de la défensive » met en relation le nombre de balles conquises et le nombre de balles perdues $(\mathrm{BC} / \mathrm{BP}$ ). II montre l'efficacité des joueurs et des joueuses à récupérer la balle (pôle de la défense) et à ne pas perdre la balle (partie défensive de l'attaque). Cet indice varie de zéro, lorsqu'aucune balle n'est conquise, à 1 si le nombre de balles conquises est égal au nombre 
de balles perdues. Si le nombre de balles conquises est supérieur au nombre de balles perdues, l'indice devient alors plus grand que 1.

\section{2. Nouveaux rôles pour l'élève}

Dans cette perspective, apprendre à lire la production des joueurs et des joueuses constitue, également, un objectif obligatoire de la formation des formateurs (Gréhaigne, Caty, \& Marle, 2004). Comme nous l'avons déjà écrit dans les rôles dévolus aux élèves celui d'observateur est particulièrement important. En effet, on ne met pas les élèves en observation pour les occuper entre deux séquences de jeu mais aussi pour apprendre. Même si certains élèves peuvent avoir besoin de supervision de la part de l'enseignant lors des premières séances (ne serait-ce que pour les rassurer), des études ont montré que des élèves aussi jeunes que 10-12 ans, peuvent, avec un minimum de pratique, recueillir des données quantitatives avec un bon niveau de précision (Godbout \& Desrosiers, 2005 ; Gréhaigne et al., 1997 ; Richard, Godbout, Tousignant, \& Gréhaigne, 1999). Les élèves observateurs peuvent faire d'autres apprentissages en observant. En plus de noter des informations prédéterminées, ils peuvent, de façon informelle, réfléchir sur l'action en cours et prendre note de comportements moteurs et/ou tactiques adoptés par leurs coéquipiers et adversaires. Cette information est doublement utile : (a) fournir une rétroaction additionnelle au cours du débat qui suivra; (b) attirer l'attention de l'observateur sur des comportements efficaces à reproduire et /ou sur des erreurs à éviter (Godbout \& Desrosiers, 2005).

Il est un autre rôle que nous allons envisager maintenant qui concerne la contribution de chaque élève à la mise au point d'actions en projet ou de feuilles de route. On définira le comportement adapté d'un élève par sa capacité à assurer deux familles articulées de fonctions: des fonctions de résolution pour élaborer et sélectionner des réponses pertinentes en vue de résoudre les problèmes formulés ; des fonctions de compréhension afin de répondre de façon cohérente et appropriée aux questions non résolues et plus généralement concevoir des actions en projet en relation avec les compétences motrices nécessaires à leur exécution.

\section{Débat d'idées}

En EPS, il ne saurait y avoir de débat véritablement scientifique entre les élèves ; aussi, nous avons préféré parler de débats d'idées (Zerai, Gréhaigne, \& Godbout, 2021) même si cette qualification peut paraître vaste et trop générale. En effet, l'un des moyens possibles 
pour accéder à la lecture interprétative d'un sujet est d'analyser les verbalisations à propos du jeu dont une modalité spécifique peut être le « débat d'idées ».

\subsection{Le déroulement du débat}

Gréhaigne et Godbout (1998b) ont proposé la définition suivante pour cette modalité d'organisation :

Situation dans laquelle les élèves explicitent (grâce à la verbalisation) et échangent des idées à propos des faits, en se fondant sur l'observation ou l'expérience personnelle. Le débat peut concerner les résultats obtenus durant l'action, le processus impliqué et ainsi de suite. (p. 114)

Le débat d'idées consiste à mettre l'élève face à une action située (ex : un match) à propos de laquelle une décision collective doit être prise. Au terme d'un affrontement préliminaire, l'élève observateur transmet à ses coéquipiers le résultat de ses observations, puis tous les membres de l'équipe échangent et confrontent différentes interprétations (les compréhensions opposées ou similaires du match, l'identification des configurations du jeu, les actions observées par rapport à celles recherchées...) et essayent de négocier des décisions communes. Ces verbalisations constituent une activité réflexive sur l'action. Ensuite, un retour à l'action permet la validation ou non des décisions formulées pendant le débat d'idées. L'objectif est de confronter les représentations avec celles présentes dans les faits observés. De ce point de vue, les programmes d'EPS en France recommandent que les élèves, face à un problème, apprennent à penser de manière critique en maîtrisant un corpus de connaissances approprié.

\subsection{Aspects cognitifs mais aussi affectifs du débat}

Dans le débat d'idées en sport collectif, il s'agit bien de proposer des séquences orales où la parole est vécue comme une interaction en vue de produire des effets cognitifs, une analyse des rapports de forces, des actions en projet ou une feuille de route. En bref, un contexte didactique où les apprenants ne se limitent pas à un rôle passif face à leur formateur détenteur du savoir. Ici, la compétence à communiquer ne renvoie pas uniquement à prendre la parole mais aussi à entendre une interrogation, faire des propositions crédibles, éviter de blesser verbalement etc. Dans un débat en EPS, la pensée critique ne devrait pas concerner seulement l'acquisition des compétences motrices car les situations offrent un contexte social concret pour explorer un plan du jeu, une nouvelle tactique ou un ensemble de règles (Poggi \& Moniotte, 2011). Au cours d'un cycle 
d'apprentissage, les élèves vont pouvoir mettre en évidence leurs succès et leurs faiblesses ainsi qu'échanger autour de conceptions et de représentations avec leurs pairs sur des actions efficaces ou non. De la sorte, les aspects affectifs ne sont pas oubliés car les élèves ont à vivre la coopération, la prise de risques dans le jeu et doivent faire preuve d'ouverture d'esprit entre autres. La durée des débats constitue une variable essentielle : pas trop longue afin d'éviter les répétitions ; pas trop courte afin de laisser le temps aux élèves de prendre une décision. Une durée de 3 à 4 minutes parait réaliste pour éviter ces deux écueils. Enfin, la plupart du temps, la place de l'enseignant reste celle d'une personne ressource (Gréhaigne, Wallian, \& Brière-Guenoun, 2015).

\subsection{Des défis pour les élèves}

Le développement du débat d'idées dans les classes s'accompagne d'un certain nombre de données qu'il va falloir prendre en compte. Pour espérer avoir un débat fructueux, il faut laisser aux élèves le temps de construire un minimum de connaissances leur permettant de raisonner sur les aspects tactiques et techniques du jeu. Pour les débutants, la grande difficulté est de nommer et mettre en mots des actions dynamiques en rapport avec des terminologies variées exprimant des notions liées à des cadres de référence théoriques fort divers (Zerai, 2011). Souvent, le premier niveau porte sur les activités collectives générales, en recourant à une langue naturelle, pour décrire les éléments organisant l'essentiel des rapports entre les joueurs et leur environnement. Puis se pose le problème de l'accès des élèves à une terminologie spécifique, pourtant passage incontournable en vue d'une communication claire et simple (Ramos et al., 2020). Ensuite, à l'aide de cette terminologie, la verbalisation a pour fonction explicite de favoriser la prise de conscience de ce qui a été fait, aidant les élèves à analyser leur propre pratique et à communiquer sur elle. A l'issue d'un débat d'idées, une feuille de route ou des actions en projet proposent une réflexion et des stratégies pour les activités à venir qui actent les décisions prises. Néanmoins, cette compétence à construire des réponses rend nécessaire de s'extraire des routines ou d'un simple constat afin de créer de nouvelles stratégies d'action.

\section{Concevoir des actions en projet : vers une feuille de route}

La feuille de route est un document à la base de tout projet. C'est elle qui vise à rassembler les facteurs d'un projet. La feuille de route s'est donc imposée comme un élément important pour accompagner tout choix de stratégie. 


\subsection{Une démarche de compréhension et de planification}

Avec ce type d'approche centrée sur l'élève où l'accent est mis sur la compréhension, une feuille de route peut se révéler très utile. Un avantage important de cette approche, parmi d'autres pour peu que la confrontation soit judicieusement choisie, est que les élèves s'approprient le problème et construisent eux-mêmes les connaissances qui y sont liées. II faut noter aussi que dans ces conditions, la mise en mots du jeu est compliquée et constitue une expérience nouvelle pour les élèves. En effet, si le joueur peut être performant au niveau des compétences motrices ou des activités cognitives recouvrant son activité en jeu, il ou elle peut éprouver de réelles difficultés concernant les termes techniques ou la verbalisation de son activité. II est vrai que de nombreuses situations anecdotiques dans les sports collectifs peuvent perturber ce processus ainsi que les caractéristiques individuelles des élèves qui ne permettent pas toujours un accès facile à la communication. Le fait de parler de projets souligne bien l'interaction joueurs / partenaires / adversaires / environnement dans toute prise de décision ou tout projet d'action qui vise à proposer une feuille de route réaliste et objectivable. Dans un débat, la mise au point d'une feuille de route ambitionne de décrire l'organisation, les consignes et les règles que doit suivre l'équipe pour atteindre les objectifs définis lors de ce débat et qui sont, bien entendu, évolutives.

\subsection{Un exemple}

Pour exemple, nous allons maintenant examiner le processus mis en place à propos d'une feuille de route élaborée après une rencontre en 5 contre 5 , sur un terrain de handball où l'équipe $\mathrm{C}$ a perdu le match sur le score de 3 - 1 avec beaucoup de pertes de balle dans la montée du ballon face à une défense en barrage.

- Sur la base de ces observations et après débat, les élèves ont planifié leur(s) action(s) : les joueurs de l'équipe $\mathrm{C}$ ont décidé de défendre devant leur but et de faire, dès la récupération du ballon, un lancer long pour une contre-attaque immédiate avec un joueur qui part très vite dans la profondeur.

- Ils ont joué de nouveau et constaté après le match que sur 5 lancers longs, ils ont marqué 3 buts et gagné la rencontre 3 - 2 .

- Après un bilan de cette planification et à partir des retours d'informations positifs, ils ont décidé de poursuivre cette stratégie de la contre-attaque avec d'autres équipes de même niveau. 
- Ainsi, en cas de nouveaux succès, ils valideront, plus avant, les règles d'action et d'organisation du jeu pour en faire des ressources stabilisées si le problème se pose à nouveau.

\subsection{Un travail de régulation socialement partagée}

Dans un débat en classe, les élèves qui y prennent part ont des avis, réflexions ou expériences différents sur le problème considéré. II s'agit donc, pour l'équipe, d'identifier, de se mettre d'accord et de décrire le périmètre du projet, la méthodologie à appliquer, d'identifier les activités à déployer et, enfin, de planifier le projet tout en y intégrant de facto une analyse critique. De la sorte, cette feuille de route dans les jeux propose, plus spécialement, le recensement des compétences motrices à mettre en œuvre, d'activités spécifiques à mener, des règles d'action et de règles de l'organisation du jeu à suivre pour réaliser les progrès envisagés en commun. Les limites pour traiter ces événements sont donc le temps, les difficultés de l'affrontement ainsi que la cohérence entre les signaux et les réponses (Gréhaigne et al., 2015).

Plus généralement, la feuille de route est une expression qui est fréquemment employée dans divers domaines pour désigner les étapes d'une stratégie ou d'un plan d'actions. En sport collectif, une feuille de route est un outil pédagogique qui acte les décisions prises lors du débat d'idées. Dans cette perspective, les joueurs sont dans l'obligation d'évaluer, d'apprendre, d'intégrer, de s'approprier de nouveaux outils sous la pression de l'évolution du jeu. Le travail d'analyse de la confrontation permet alors, avec ses partenaires, de coconstruire des contenus répondant aux exigences d'un jeu pertinent, efficace et performant. Suivant le niveau et les habitudes des élèves, c'est un document écrit, qui détaille de manière plus ou moins précise la ou les tâches pour que l'équipe progresse. Pour mieux la faire fonctionner, Billard (2005) propose concrètement une observation participante. Ainsi, il suggère, pendant un temps défini, que des observateurs recueillent des informations sur les deux équipes en présence. Lors du premier arrêt de jeu, les observateurs communiquent les informations à leur équipe sur leur propre production ainsi que sur l'organisation de l'équipe adverse. Le groupe enregistre ces informations, les met en relation et prend des décisions pour la séquence à venir. Par exemple, une équipe qui a des difficultés avec la circulation du ballon peut décider temporairement de tenter de laisser la balle aux adversaires pour jouer le contre et tirer le plus vite possible au but. Cette décision doit devenir quantitativement observable (augmentation du nombre de buts par exemple) afin d'objectiver la stratégie et ainsi apprécier l'adéquation entre les décisions prises et le jeu 
produit pendant la séquence. Ici, l'évaluation formative aide les élèves à apprendre et à développer leurs ressources. L'observation formative peut être instrumentée ou purement intuitive mais si on désire vraiment obtenir une observation de qualité, rigoureuse et systématique, il faut s'en donner les moyens afin de rendre plus tangibles les implications pédagogiques de l'évaluation.

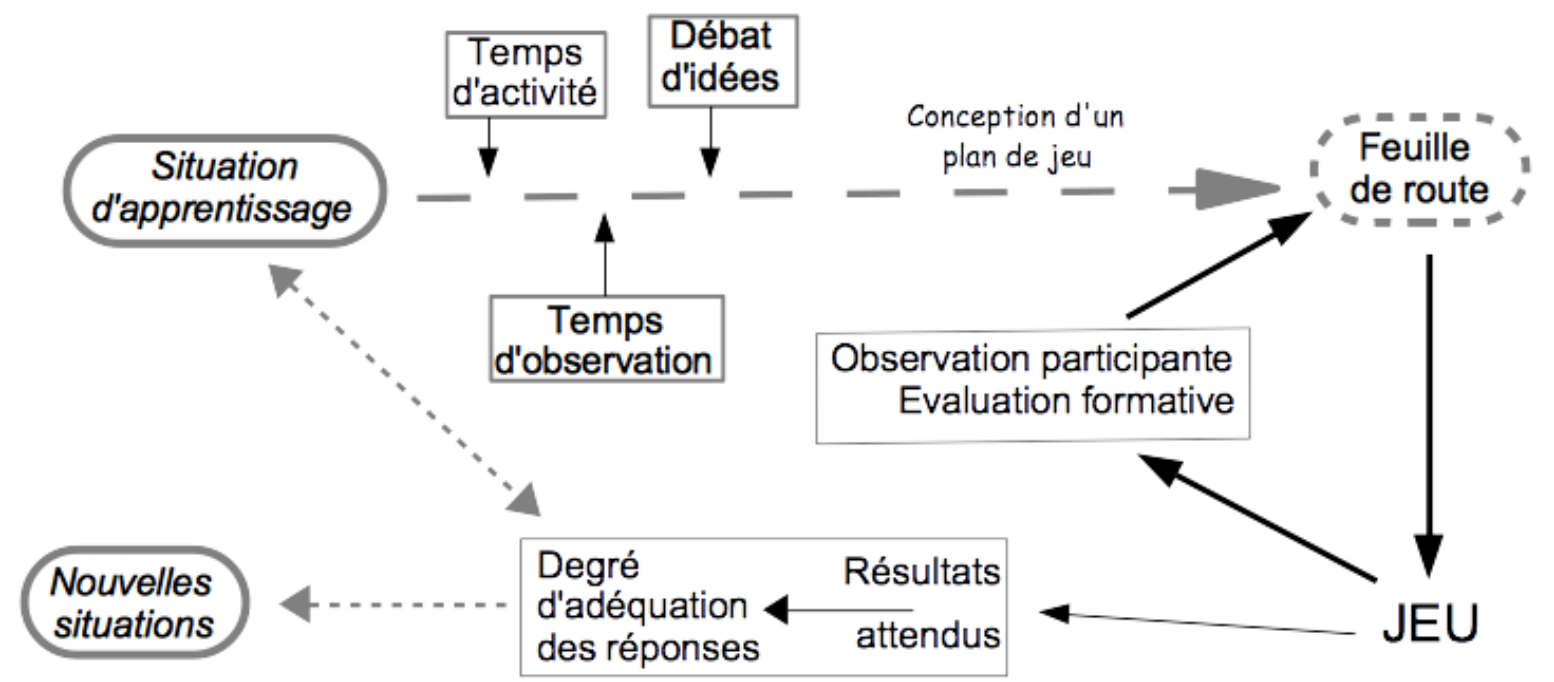

Figure 2. Différentes facettes de l'observation et de l'évaluation formative de l'activité des élèves dans le jeu (adaptée de Gréhaigne et al., 2015).

La figure 2 présente différents aspects de l'observation avec les sports collectifs qui peuvent se dérouler pendant ou après la rencontre. Cependant, avant de s'engager dans ces situations, les élèves doivent savoir ce que l'on attend d'eux et/ou avoir décidé ce qu'ils ont à faire. Les élèves et l'enseignant doivent savoir jusqu'à quel point la tâche est réussie ou non, et en particulier ce qui est positif et ce qui ne l'est pas. II faut donc recueillir des informations fiables et fidèles sur les performances qui seront utiles pour planifier les prochains matchs. II est à noter que pour Allal, Cardinet et Perrenoud (1979), la régulation est le concept central de l'évaluation, dans le sens où toute évaluation, explicitement ou implicitement, est un moyen de régulation à l'intérieur d'un système de formation.

Par ailleurs, les premières expérimentations montrent l'importance de l'engagement des élèves dans ce type de travail. Il est nécessaire de considérer que si le jeu n'a pas un enjeu significatif pour les élèves et s'ils ne s'inscrivent pas dans un projet commun, les apprentissages sont vraiment faibles voire absents. A l'opposé, si l'enjeu proposé constitue 
un pas en avant trop important, les élèves décrochent et ne veulent plus participer. Chez certains élèves en difficulté, la perception initiale forge une représentation anticipée des exigences de la situation qui se confirme ou non lors du jeu. II s'agit d'un aspect important de la dynamique de la leçon car cette perception peut affecter la manière d'aborder les débats à venir et les confrontations ultérieures. Mais, le professeur n'est pas absent du système enseignement / apprentissage et il peut apporter des correctifs afin d'accroitre la motivation et de faciliter les apprentissages. L'enseignant voit le jeu, les réussites et les erreurs et peut décider de fournir des informations à moins qu'il préfère laisser les élèves réfléchir à leurs actions à la lumière des différents retours d'informations qu'ils ont reçus.

Dans un contexte d'apprentissage collaboratif, la régulation peut prendre trois formes: l'autorégulation, la co-régulation, la régulation partagée ou socialement partagée. Järvelä, Järvenoja, Malmberg, et Hadwin (2013) ont écrit :

... l'autorégulation survient lors de tâches indépendantes, coopératives ou collaboratives et conduit à des changements dans les connaissances, les croyances et les stratégies que les individus transposent dans des contextes de nouvelles tâches et dans des changements en termes de structures et de conditions de l'environnement. (p. 269)

Selon Hadwin, Järvelä et Miller (2018), des perspectives d'apprentissage situé ont étendu plus récemment les théories et modèles d'autorégulation à des situations d'apprentissage hautement interactives et dynamiques où émergent la construction partagée du savoir et la collaboration. L'autorégulation de l'apprentissage est devenue la pierre angulaire pour l'exploration de formes de régulation plus sociales telles que la co-régulation et la régulation partagée ou socialement partagée. Selon Allal (2016), la co-régulation résulte de l'influence conjointe (a) de l'autorégulation de l'élève et (b) de la régulation provenant d'une autre source (en particulier de l'enseignant, d'un pair, d'une procédure et d'instruments d'évaluation) sur la progression des apprentissages de l'élève. Finalement, pour Järvelä et al. (2013), la régulation socialement partagée de l'apprentissage réfère aux processus par lesquels les membres d'un groupe régulent leur activité collective. Ce type de régulation implique des processus régulateurs interdépendants ou collectivement partagés, des croyances et des connaissances (e. g., des stratégies, de la surveillance, de l'évaluation, de la détermination d'objectifs, de la motivation, des prises de décision métacognitives) orchestrés au service de résultats co-construits ou partagés. Cette vision de la régulation reflète bien, à notre avis, l'approche préconisée par le MADT. Les composantes observation, débat, feuille de route, itérations sont partie prenante du processus selon leurs 
mécanismes spécifiques mais toutes sont axées sur les mêmes buts : informer l'équipe, lui permettre de réfléchir sur l'expérience de jeu précédente, d'identifier des points forts et des points faibles, de se construire un projet d'action conséquent, et de vérifier l'à propos de ce projet lors de situations de jeu subséquentes.

\section{Quelques aspects théoriques qui fondent cette approche}

Cette conception de l'enseignement des sports collectifs incorpore et repose sur un certain nombre d'aspects théoriques sur lesquels nous allons revenir maintenant. Ce choix de l'incorporation de connaissances s'appuie sur l'idée qu'un croisement entre plusieurs approches de la réalité évite les écueils des relations traditionnelles entre théorie et pratique: une pratique qui est abordée sans base théorique et/ou des théories qui demeurent plus ou moins coupées de la pratique.

\subsection{Les différentes formes de pensée au service de l'action et du jeu}

L'intelligence de jeu dans les sports d'équipe est généralement considérée comme quelque chose de peu appréhendable et les excellents joueurs sont souvent caractérisés par leur bonne «lecture du jeu». La plupart des formateurs sont d'accord sur ce qui constitue de bonnes compétences motrices (technique, force, agilité, endurance, etc.) ; par contre, ce qui caractérise un bon joueur en termes d'intelligence de jeu est moins évident. C'est là que la construction d'une pensée opérationnelle intervient. Au sens large, la pensée est une activité psychique, consciente dans son ensemble, qui recouvre les processus par lesquels sont élaborés, en réponse aux perceptions venues des sens, des images, des sensations ou des concepts ayant la connaissance pour objet et que les joueurs manipulent et associent pour apprendre, créer et agir. (cf. CNRTL, 2018).

\subsubsection{Pensée critique}

La pensée critique est complexe et, parfois, représente un processus insaisissable qui nécessite une solide base de connaissances en vue d'une bonne compréhension de ses apports. Les mécanismes de la pensée permettent au joueur d'être au contact de son environnement pour élaborer des concepts et les relier entre eux afin d'apprendre, de créer et d'agir. Cependant, trop souvent en sport collectif, dans la formation tactique des joueurs, le décodage des configurations du jeu est assimilé à un ensemble de recettes. Ce type de formation est en opposition avec une conception de l'apprentissage où le recours aux mécanismes de métacognition permet au joueur non seulement d'évaluer et d'interpréter 
s'il est en situation de réussite ou d'échec mais également de concourir à ses propres transformations (Godbout, 2003 ; Godbout \& Gréhaigne, 2020).

Le recours à la pensée critique n'est pas récent en éducation physique (Schwager \& Labate, 1993 ; Tishman \& Perkins, 1995). Cependant, il existe de nombreuses façons de définir la pensée critique. Nous avons choisi celle de McBride (1991) qui postule que « la pensée critique en éducation physique doit être définie comme une pensée réflexive utilisée pour prendre des décisions raisonnables et défendables sur les tâches ou les défis liés au mouvement» (p. 115). Nous pourrions ajouter que cette présentation implique un raisonnement de type cybernétique particulièrement causal et évaluatif -voire normatif ainsi qu'une réflexion sur la planification et les algorithmes stratégiques possibles. Or la performance physique efficace implique le raisonnement, la réflexion, l'élaboration de stratégies et la planification, toutes parties du processus de pensée critique, mais également l'intuition, le dialogue avec autrui, l'interprétation d'une situation en évolution, voire la prise en charge du hasard. Alors que dans la forme classique du débat les élèves ont recours à une pensée critique d'opposition (certains argumentant en faveur et d'autres contre une résolution donnée), le débat d'idées préconisé ici fait appel à une pensée critique coopérative qui vise à réconcilier la position des uns et des autres, à co-construire à partir des idées de chacun de sorte que chacun bénéficie de l'expérience (Tan, 2017). Dans le contexte du débat, cela suppose le recours à des habiletés cognitives telles que l'interprétation, l'argumentation, l'inférence, l'analyse, l'évaluation, la mise à l'avant de solutions, la création, et la prise de décision selon le contexte. Cela suppose également de rechercher de l'information signifiante et fiable et de pouvoir s'adapter aux changements (Uribe Enciso, Uribe Enciso, \& Vargas-Daza, 2017).

\subsubsection{Pensée réflexive sur le jeu et dans le jeu}

La pensée réflexive (Pallascio \& Lafortune, 2000) s'appuie principalement sur la notion de prise de conscience de ses propres connaissances et de ses expériences passées car, souvent, celles-ci recèlent des informations nécessaires aux prises de décision actuelles. La pensée réflexive, qu'elle soit sur le jeu antérieur (on action) ou dans le jeu concomitant (in action), est une partie du processus de la pensée critique et n'est pas uniquement destinée à des réflexions épistémologiques, mais aussi et surtout à des utilisations dans des situations réelles et concrètes. Dans le jeu, la conceptualisation est bien au service de l'action en cours, en vue d'une théorisation à court terme qui offre au joueur une « structure conceptuelle de la situation » (Vidal-Gomel \& Rogalski, 2007). Celle-ci utilise les relations causales entre invariants opératoires, règles d'action, règles de l'organisation du jeu et 
compétences motrices en relation avec les indicateurs de prise d'information ou de décision. Cette structure conceptuelle permet aux élèves ou aux joueurs de construire une représentation mentale contextualisée de la situation, orientée vers l'action qui se déroule et se transforme avec le temps au fil des réinterprétations du jeu.

Quant à elle, la pratique réflexive sur le jeu est une activité qui consiste à caractériser la manière dont l'action s'est organisée, à lire et interpréter ce qui sous-tend l'action et à mettre en mots ses connaissances pour les autres. Plus précisément, le débat d'idées aide les apprenants à développer une pensée réflexive et critique en examinant des arguments contradictoires et variés et en collectant des informations signifiantes ou non pour l'action. Ensuite, ils peuvent effectuer des analyses pour hiérarchiser les décisions plausibles au regard de leurs effets, remettre en question des projets en évaluant les résultats de l'action. Le développement d'une pensée et d'une pratique réflexive s'articule notamment autour d'une pensée critique et créative, de compétences argumentatives et d'habiletés métacognitives (Godbout, 2003).

\subsubsection{Pensée tactique}

Enfin, la pensée tactique est basée sur l'expérience et des outils cognitifs qui lui confèrent un caractère actif, favorisant l'initiative. Dans les sports collectifs, le développement d'une pensée tactique autonome (Gréhaigne \& Caty, 2014 ; Menaut, 1998) permet d'analyser et d'organiser les réponses face à des problèmes analogues. La complexité dans les jeux d'opposition requiert stratégie et tactique. II n'y a que la tactique pour apprivoiser l'incertain voire l'aléatoire. Elle consiste en l'art d'utiliser les informations qui surviennent dans l'action, de les intégrer, de formuler des réponses au fil du jeu et d'être apte à rassembler le maximum d'éléments fiables pour affronter les transformations des configurations momentanées. La pensée tactique est donc essentiellement la pensée qui traite avec l'incertitude et qui est capable de regrouper et de relier des indices dans un tout en sachant reconnaître le singulier. La possibilité de progrès repose précisément sur la reconnaissance du rôle déterminant, parce que première chez l'être humain, de la pensée organisatrice abstraite de l'action, c'est-à-dire, dans le jeu, de la pensée tactique. Bien sûr, celle-ci doit être considérée aussi dans une unité dialectique avec la technique. Mais c'est la pensée qui donne un contenu à l'utilisation des procédés techniques en les transformant en actions.

Cette forme d'intelligence engagée dans le jeu doit combiner à la fois le flair et l'intuition, l'attention vigilante et le sens de l'à-propos. En situation de confrontation, l'accent est toujours mis sur «l'efficacité pratique », en un mot la recherche de la réussite dans le domaine concret de l'action. Tout acte de jeu est forcément tactique, quel que soit le niveau 
du joueur, car il consiste à résoudre, pratiquement et dans le respect du règlement, les problèmes posés par les rapports d'opposition : expérience, délibération et initiative sont bien au cœur de la pensée tactique.

Ces apprentissages s'effectuent à l'aide d'exercices appropriés en situation d'opposition et visent à éviter aux élèves d'apprendre à jouer de façon schématique. La capacité à observer, caractériser et rendre compte des évènements de jeu devient cruciale. En effet, à y regarder de plus près, une approche qui proposerait une solution technique, sans aller à la racine du problème, risquerait fort de ne traiter que des effets secondaires sans atteindre les causes réelles du problème généré par l'opposition. La pensée technique qui consiste à décomposer un problème et à lui attribuer une solution technique et une seule est, ici, inopérante car elle relève d'une pensée purement empirique fondée sur une expérience étriquée.

Le passage de la pensée à l'action et de l'action à la pensée relève de processus complexes et dynamiques qui sont difficiles à appréhender (Memmert \& Roth, 2007 ; Gréhaigne \& Godbout, 2014). En définitive, la pensée est cette faculté originale qui permet à l'homme de ne plus être prisonnier du monde perçu et donc de ses organes des sens. En effet, la pensée permet de prendre du recul par rapport aux informations délivrées par nos perceptions et autorise la conception d'autres univers par opposition au seul monde perçu (Ochanine, 1978). Dans ce cas, Mouchet, Amans-Passaga et Gréhaigne (2010) proposent de distinguer différents niveaux d'abstraction qui consistent d'abord à (a) réfléchir collectivement sur les données concrètes de la situation en liaison étroite avec la perception du jeu, (b) utiliser la pensée tactique liée au jeu mais dépassant cette situation concrète pour enfin (c) recourir à une pensée tactique abstraite qui n'est pas directement liée à l'activité présente. Débattre d'une idée à propos de l'action devient ainsi un moyen de s'affranchir de l'immédiateté des actions, des émotions et de la masse des informations. II s'agit de prendre de la distance par la formalisation de l'action dans la pensée, par le choix des règles de l'action efficace et par l'adoption d'un plan d'action construit avec ses pairs au travers du langage (Wallian \& Gréhaigne, 2004). Ainsi, passer d'une analyse spontanée des configurations du jeu à une caractérisation qualitative de celles-ci exige une analyse des actions qui tienne compte du rapport de force. Les aspects perceptifs s'en trouvent complexifiés et la pensée tactique doit être en mesure de faciliter et de conforter ces processus. 


\section{2. Le constructivisme}

Le constructivisme est une théorie de l'apprentissage, qui a été développée, entre autres, par Piaget (1923), en réponse au béhaviorisme qui, d'après lui, limitait trop l'apprentissage à une association stimulus-réponse, le sujet étant considéré comme une boite noire. L'approche constructiviste s'intéresse à l'activité du sujet pour se construire une représentation de la réalité qui l'entoure. Le constructivisme part de l'idée que les connaissances de chaque sujet ne sont pas spécialement une copie de la réalité, mais un modèle plus ou moins fidèle de celle-ci construit par lui au cours du temps. Le constructivisme s'attache à étudier les mécanismes et processus permettant la construction de ce modèle chez les sujets à partir d'éléments déjà intégrés. On est donc à l'opposé d'une simple approche de type boite noire.

La compréhension, constamment renouvelée, s'élabore à partir des représentations plus anciennes d'événements passés, que le sujet restructure en interne, à partir des informations reçues en regard de ses connaissances et de ses expériences. L'apprentissage n'intervient que dans une structure d'accueil déjà constituée (Ennis, 2007) qui sert de base à la lecture et à l'interprétation des situations didactiques. L'activité d'apprentissage peut être définie comme une activité de construction de sens, dans laquelle le sujet réorganise son horizon d'attentes (Wallian, Poggi, \& Chauvin-Vileno, 2008 ; Genevois \& Wallian, 2020). La théorie constructiviste des apprentissages (Piaget, 1923, 1969 ; Vygotski, 1985) considère que c'est dans l'interaction avec l'environnement que le sujet élabore les stratégies adaptatives. En construisant sa connaissance, le sujet passe d'un système interprétatif à un autre.

\section{3. Du constructivisme au socio-sémio-constructivisme}

Dans cette perspective sémio-constructiviste (Wallian \& Chang, 2007 ; Wallian \& Gréhaigne, 2004), la difficulté consiste à accéder à la manière dont l'élève s'y prend pour apprendre et à comprendre comment s'opère le dialogue entre pensée et action. Les élèves sont amenés à construire, dans des jeux à effectif réduit, leurs compétences tactiques et motrices en les adaptant individuellement selon la situation spécifique vécue. Une telle option soutient que les connaissances construites par l'élève sont le résultat de l'interaction entre son activité cognitive et la réalité où « apprendre au travers de la compréhension » devient une réalité élaborée dans le dialogue avec autrui dans l'affrontement.

II s'agit, entre autres, à l'aide d'une problématique radicalement interactionniste, de chercher à saisir dans les faits langagiers les traces des conduites signifiantes produites en jeu. II 
convient, ici, d'envisager l'interaction socio-discursive comme un processus complexe à la fois mental, social et langagier (Bronckart, 1996).

En sports collectifs, le décryptage des situations de jeu avec leurs configurations momentanées ou prototypiques constitue un support intéressant pour le débat d'idées (Gréhaigne, Caty, Billard, \& Chateau, 2005). Les premiers débats, dépourvus de spécificité technique, laissent progressivement place à un discours descriptif et argumentatif. Chaque pratiquant redéfinit cet objet au regard de ce qu'il connaît, de ce qu'il croit connaître et comprendre et de ce qu'il a envie de faire. Nous visons donc au travers du débat d'idées une auto-socio-construction des compétences motrices et connaissances tactiques avec une approche que nous qualifierons, dans ce cadre, de « socio-sémio-constructiviste ». Cette conception de l'apprentissage au sein d'une activité interprétative incarnée permet de déployer chez les élèves une activité de problématisation. II s'agit, ici, d'établir une relation dialectique entre le connu et l'inconnu, entre le projeté et le constaté, en spéculant sur la nature d'un problème rencontré dans le jeu. En sport collectif, la problématisation s'oriente vers la recherche d'une réponse transitoire au problème présent qui va surgir de l'interaction sujets / rapport d'opposition. Pour le joueur, en actes, cela nous renvoie bien à une démarche d'auto-socio-construction soulignant ici que l'acte d'apprendre est un acte singulier, individuel (on n'apprend rien au joueur, c'est toujours lui qui apprend ou pas) mais que cet apprentissage se conduit dans un cadre socialisé.

\subsection{Processus cognitifs, conscientisation et/ou mentalisation}

Sommes-nous bien conscients des processus mentaux engagés ou sommes-nous incapables d'y avoir accès étant donnée la transparence des phénomènes d'interprétation et de prise de décisions ? La conscientisation possède, certes, ses propres limites mais une bonne partie de la cognition reste à explorer. Conscientisation, explicitation et compréhension sont des processus mentaux qui visent à apprendre à partir d'expériences vécues, par un retour de la conscience sur son propre fonctionnement (Deleplace, 1983 ; Freire, 1974). L'objectif éducatif est de prendre conscience de ses manières d'agir et de réagir, dans les situations de la vie. Le recours, entre autres, aux processus cognitifs conscients dans la gestion de certains aspects du jeu et de l'apprentissage a entraîné une profonde modification de la conception des apprentissages en sport collectif. La mentalisation est un processus mental par lequel une personne prend conscience d'un phénomène ou des représentations intellectuelles de ce phénomène, voire interprète ses propres comportements et ceux d'autrui, sur la base d'états intentionnels (CNTRL, 2020). 
Même si elle reste discutée par les tenants des apprentissages moteurs, cette "mentalisation" ou « conscientisation " selon les auteurs s'effectue à partir du fonctionnement effectif de l'organisation du jeu. Dans le jeu, tout s'appuie sur la perception consciente et systématique des mouvements tactiques des adversaires, quelle que soit l'équipe qui détient momentanément l'initiative par rapport aux configurations en cours.

On peut supposer que face au système complexe que constituent le jeu et ses configurations, le joueur a besoin d'automatiser un certain nombre d'éléments afin de répondre plus vite aux problèmes posés par l'affrontement. Pour cela, il est nécessaire de formuler l'hypothèse que l'expérience contribue à automatiser certains aspects de la pratique du joueur, par la mise en place de routines et d'un système d'inter-régulations de ces routines. II faut donc permettre à l'élève d'automatiser peu à peu certaines connaissances et la régulation des compétences motrices vers des structures infraconscientes, ce qui libère des ressources attentionnelles pour l'analyse du jeu en cours.

Néanmoins, si un échec interrompt temporairement la continuité de l'activité, alors le joueur sera obligé de redéfinir son action en cours, donc de revenir à l'activité réfléchie pour permettre de trouver une solution à cet échec. II est à noter que la tactique tient un rôle central dans l'activité cognitive et motrice des joueurs impliqués dans un rapport de force. En ce qui concerne les compétences motrices, on vise, le plus souvent, une automatisation partielle de celles-ci afin de dégager le canal cognitif conscient pour assurer d'autres opérations. Cela consiste, par exemple, à faire réguler, sauf alerte, au niveau de processus infra conscients, un certain nombre de gestes techniques offrant de ce fait la possibilité pour d'autres programmes de fonctionner en même temps. Le recours aux processus cognitifs, conscients ou infra conscients, prend toute sa valeur lors de la confrontation des élèves à des situations problèmes les amenant à mettre en œuvre des stratégies de résolution de ces problèmes. De surcroit, être conscients des apprentissages réalisés permet aussi d'aboutir à la construction de compétences motrices qui ne sont pas isolées du contexte.

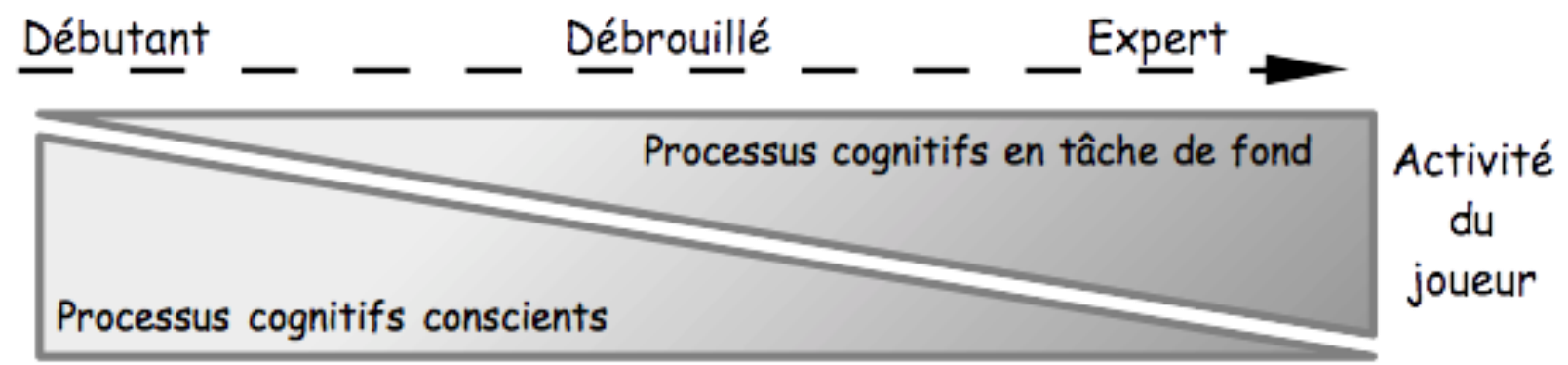

Figure 3. Évolution des processus cognitifs en fonction de l'expertise. 
La figure 3 illustre la façon dont s'opère l'évolution d'un débutant encombré par une multitude d'informations vers un joueur de bon niveau qui ne relève que les quelques indices pertinents, voire qui apporte une réponse sans avoir, apparemment, relevé d'indices. Lors de ces opérations, la part des processus conscients diminue au profit du traitement d'autres informations de hiérarchie plus élevée.

\section{Conclusion}

Tout type d'apprentissage basé sur l'activité interprétative et décisionnelle du joueur prend en charge ses interlocutions, ses observations et sa capacité à transformer ses propres réponses pour agir sur son réel du jeu. Cependant, la portée véritable du débat d'idées pour le plus grand nombre reste encore à explorer et à valider. Le " métier » d'élève correspond certes aux comportements et compétences attendus par l'institution scolaire en vue de produire une performance scolaire : toutefois, il est inscrit dans un système d'interactions avec d'autres acteurs que sont l'enseignant et le groupe classe. Habituellement, un élève qui effectue bien son métier maîtrise à peu près les rituels, les règles, les gestes, les outils, les connaissances, toutes ces petites choses qui font le bon élève. Par contre, le très bon élève ou le très mauvais élève sortent de la « norme » : que pensent-ils de cette nouvelle démarche d'apprentissage ? En tirent-ils plus ou moins profit que leurs camarades de niveau scolaire différent? Certains bons élèves, très à l'aise dans le système scolaire, regimbent et refusent de participer au débat d'idées ainsi qu'aux tâches d'observation ou d'arbitrage, considérant que c'est du « temps perdu » dans les apprentissages dits moteurs. Ils argumentent auprès du professeur en lui disant « dites-moi ce qu'il faut faire pour apprendre car c'est votre job ». Cette attitude est peu courante mais elle existe (Caty, Zerai, \& Gréhaigne, 2009). Ou encore, à l'inverse, ils peuvent être les destinataires exclusifs et privilégiés de la démarche du fait de l'ensemble des dispositions familiales les familiarisant précocement de façon continue à la réflexivité (Johsua \& Lahire, 1999). Pour d'autres, le métier de l'élève consiste simplement à empêcher le maître de faire le sien, non par goût systématique de la contestation, mais pour résister aux demandes de l'école parfois par trop éloignées de leurs représentations. Cependant, on peut observer que les qualités requises pour observer et rendre compte du jeu permettent parfois de valoriser des élèves qui rencontrent des difficultés dans les situations d'affrontement au motif qu'ils n'y comprennent rien.

Dans ce type d'approche didactique qui donne la parole aux élèves, une des difficultés majeures rencontrées concernant le métier d'enseignant est, pour le professeur, de rester 
suffisamment neutre pour laisser les élèves interpréter, s'exprimer et explorer des solutions. Mais également de veiller à ce que les élèves les moins enclins à prendre la parole, les filles et les enfants issus des milieux moins favorisés, interviennent dans le débat afin que les profits de la démarche soient également partagés. La dévolution du problème aux élèves permet à la majorité de ceux-ci de prendre à bras le corps la perspective d'apprentissages authentiques. En fin de compte, former les élèves à ces nouveaux aspects du métier d'élève devrait les aider à devenir progressivement des acteurs de leur apprentissage tout en étendant la marge d'initiative : cette approche leur permettrait à terme une forme d'émancipation dans l'interprétation et la prise de décision au quotidien.

Cette notion de liberté d'initiative revêt une importance nouvelle dans ce modèle d'apprentissage. II s'agit de mobiliser les capacités adaptatives à la fois de l'intelligence et de l'action. Cela permet d'éviter de rester prisonnier des seules modalités les plus couramment utilisées que sont l'application et la répétition. Par conséquent, il faut toujours chercher à mobiliser et à développer une autre modalité : la création d’opportunités dans le jeu.

Les auteurs remercient les experts pour leur lecture attentive et leurs propositions à propos d'une première version de cet article.

\section{Bibliographie}

Alin, C., Bourrier, J.-C., Buono, A., Jade, R., \& Martinez, E. (1979). Football, l'enfant et l'activité sportive. Mémento football du CPS-FSGT. Paris : Sport et plein air.

Allal, L. (2016). The co-regulation of student learning in an assessment for learning culture. In: D. Laveault \& L. Allal (Éds.), Assessment for learning: Meeting the challenge of implementation (pp. 259-273). The Enabling Power of Assessment (volume 4). Cham, Switzerland: Springer.

Allal, L., Cadinet, J., \& Perrenoud, P. (Éds.). (1979). L'évaluation formative dans un enseignement différencié. Berne : Peter Lang.

Amicale des Anciens Élèves de l'ENSEPS (1966). Des colloques de Vichy 1964 - 1965. Éducation Physique et Sport, 78, 19-73.

Billard, M. (2005). La feuille de route. Notes de cours (non publiées), Université de Bourgogne. Centre Condorcet (STAPS), Le Creusot.

Bouthier, D. (1988). Les conditions cognitives de la formation d'actions sportives collectives (Thèse non publiée). Université Paris V, EPHE, Paris. 
Brau-Antony, S., \& Cleuziou J. P. (2005). L'évaluation en EPS. Paris : Actio

Bronckart, J.-P. (1996). Activité langagière, textes et discours. Pour un interactionnisme socio-discursif. Lausanne : Delachaux \& Niestlé.

Brousseau G. (1986). Fondements et méthodes de la didactique des mathématiques. Recherches en Didactique des Mathématiques, 7(2), 33-115.

Caty, D., Zerai, Z., \& Gréhaigne, J.-F. (2009). Un cycle de handball au lycée de Gray et de Thala. In J.-F. Gréhaigne (Éd.), Autour du temps. Apprentissages, espaces, projets dans les sports collectifs (pp.189-228). Besançon : Presses de l'Université de Franche-Comté.

Centre national de ressources textuelles et lexicales (2018). Pensée. Consulté sur : http://www.cnrtl.fr/definition/pensée.

Centre national de ressources textuelles et lexicales (2020). Mentalisation. Consulté sur : http://www.cnrtl.fr/definition/modélisation.

Chandler, T. (1996). Teaching games for understanding - Reflections and further questions. Journal of Physical Education, Recreation \& Dance, 67(4), 49-51.

Chang, C.-W. (2009). Langage, pensée et action : Approche sémio-constructiviste des apprentissages du jeu en basket-ball chez l'élève de CM2 (5ième grade) (Thèse non publiée). Université de Franche-Comté, Besançon (France).

Darnis, F., Lafont, L., Menaut, A. (2007). Interactions verbales en situation de coconstruction de règles d'action au hand-ball: L'exemple de deux dyades à fonctionnement contrasté. eJRIEPS, 11, 56-17.

Deleplace, R. (1966). Le rugby. Paris : Colin - Bourrelier.

Deleplace, R. (1979). Rugby de mouvement-Rugby total. Paris : Éducation Physique et Sport.

Deleplace, R. (1983). La recherche sur la spécialisation sportive, l'entraînement, la performance. Actes du Colloque la recherche en STAPS. Nice, 19-20 septembre, 93-151.

Deriaz, D., Poussin, B., \& Gréhaigne, J-F. (1998). Le débat d'idées. Revue EP\&S, 273, 8082.

Ennis, C. D. (2007). Curricular coherence: A key to effective physical activity programs. In P. Heikinaro-Johansson, R. Telama \& E. McEvoy (Éds.), The role of physical education and sport in promoting physical activity and health (pp. 10-25). Proceedings of the 2006 AIESEP Congress, 05-08 July 2006 Jyväskilä, Finland. 
Freire, P. (1974). La pédagogie des opprimés (suivi de Conscientisation et révolution). Paris : Maspero.

Genevois, S., \& Wallian, N. (Éds.). (2020). La didactique en tous terrains. De la didactique contextualisée à la contextualisation du didactique. Paris : Éditions Archives Contemporaines.

Godbout, P. (2003). Reflection on innovation and renovation: Authentic assessment and subject matter expertise in Physical Education. In L. Sena Lino, R. Trindade Ornelas, F. Carreiro Da Costa, \& M. Piéron (Éds.), Innovations and new technologies in physical education, sport, research and/on teacher and coach preparation (Cagigal Lecture). Proceedings CD-ROM of the AIESEP Congress, University of Madeira, 22-25 November 2001.

Godbout, P., \& Desrosiers. P. (2005). Students' participation to the assessment process in physical education. In F. Carreiro da Costa, M. Cloes, \& M. Gonzalez Valeiro (Éds.), The art and science of teaching in physical education and sport (pp. 227248). Lisboa, Portugal: Faculdade de Motricidade Humana, Universidade Tecnica de Lisboa.

Godbout, P., \& Gréhaigne, J.-F. (2020). Revisiting the Tactical-Decision Learning Model. Quest. 72, 430-447.

Gréhaigne, J.-F. (1989). Football de mouvement. Vers une approche systémique du jeu. Thèse de Doctorat non publiée. Université de Bourgogne, Dijon.

Gréhaigne, J.F. (1992). L'organisation du jeu en football. Paris : ACTIO.

Gréhaigne, J.-F. (1995). Quelques données de recherche sur les aspects tactiques dans les sports collectifs. In C.-A. Hauert, L. Bizzini, J. Brechbûhl (Éds.), Pages choisies de sciences du sport : Psychologie - Tennis (pp. 61-83). Genève : Université de Genève, Médecine et Hygiène.

Gréhaigne, J.-F. (Ed.). (2016). Les objets de la technique. De la compétence motrice à la tactique individuelle. Besançon: Presses de l'Université de Franche-Comté.

Gréhaigne, J.-F. (2019). Utiliser les sports collectifs pour enseigner et apprendre en EPS.

Notes de cours. Berlin : Éditions Universitaires Européennes.

Gréhaigne, J.-F., Billard, M., Guillon, R., \& Roche, J. (1989). Vers une autre conception de l'enseignement des sports collectifs. In G. Bui-Xuan, P. Boyer, P. Chifflet \& Y. Leziart (Éds.), Méthodologie et didactique de l'éducation physique et sportive (pp. 201-216). Paris : Association Francophone pour la Recherche en Activités Physiques et Sportives. 
Gréhaigne, J.-F., Billard, M., \& Laroche, J.-Y. (1999). L'enseignement des jeux sportifs collectifs à l'école. Conception, construction, évaluation. Bruxelles : De Boeck.

Gréhaigne, J.-F., \& Caty, D. (2014). Développer la pensée tactique à l'école. In J.-F. Gréhaigne (Éd.), L'intelligence tactique. Des perceptions aux décisions tactiques en sports collectifs (pp. 279-299). Besançon : Presses de l'Université de FrancheComté.

Gréhaigne, J.-F., Caty, D., Billard, M., \& Chateau, L. (2005). Les concepts de matrice offensive et défensive envisagés sous l'angle de la didactique des sports collectifs. eJRIEPS, 7, 23-47.

Gréhaigne, J.-F., Caty, D., \& Godbout, P. (2010). Modelling ball circulation in invasion team sports: A way to promote learning games through understanding. Physical Education and Sport Pedagogy, 15, 257-270.

Gréhaigne, J.-F., Caty, D., \& Marle, P. (2004). L'apport de la notion de configuration du jeu à la didactique des sports collectifs. In G. Carlier (Éd.), Si l'on parlait du plaisir d'enseigner l'éducation physique (pp. 167-179). Montpellier: AFRAPS.

Gréhaigne, J.-F., \& Godbout, P. (1995). Tactical knowledge in team sports from a constructivist and cognitivist perspective. Quest, 47, 490-505.

Gréghaigne, J.-F. \& Godbout, P. (1998a). Formative assessment in a tactical approach context. JOPERD, 69(1) 46-51.

Gréhaigne, J.-F., \& Godbout, P. (1998b). Observation, critical thinking and transformation: three key elements for a constructivist perspective of the learning process in team sport. In Feingold, R., Roger Rees, G. Barette, L., Fiorentino, S. Virgilio, \& E. Kowalski (Eds.), Education for life (pp. 109-118). New York: Adelphi University Press.

Gréhaigne, J.-F., \& Godbout, P. (2014). Dynamic systems theory and team sport coaching. Quest, 66, 96-116.

Gréhaigne, J.-F., Godbout, P., \& Bouthier, D. (1997) Performance assessment in team sport. Journal of Teaching in Physical Education, 16, 500-516.

Gréhaigne, J.-F., Godbout, P., \& Bouthier, D. (1999). The foundations of tactics and strategy in team sports. Journal of Teaching in Physical Education, 18, 159-174.

Gréhaigne, J.-F., Godbout, P., \& Bouthier, D. (2001). The teaching and learning of decision making in team sports. Quest, 53, 59-76.

Gréhaigne, J.-F., Godbout, P., \& Mahut, N. (1999). L'enseignement des jeux par la compréhension : une revue de questions. STAPS, 48, 81-93. 
Gréhaigne, J-F., Mahut, N., \& Godbout, P. (2000). Observation du jeu : nouvelle donne dans le métier d'élève. Revue EP\&S, 285, 71-74.

Gréhaigne, J.-F., \& Marle, P. (2016). Histoire et actualité de l'évolution de l'observation et de l'évaluation des techniques. In J.-F. Gréhaigne (Éd.), Les objets de la technique - De la compétence motrice à la tactique individuelle (pp. 87-107). Besançon : Presses de l'Université de Franche-Comté.

Gréhaigne, J-F., Poggi M.-P., \& Zerai, Z. (2017). L'enseignement et l'apprentissage des connaissances et des compétences motrices utiles en sport collectif. eJRIEPS, 40, 163-184.

Gréhaigne, J.-F., Wallian, N., \& Brière-Guenoun, F. (2015). Débat d'idées, langage et interactions discursives et apprentissage des sports collectifs. eJRIEPS, HS $n^{\circ}$, 54-78.

Gréhaigne, J.-F., Wallian, N., \& Godbout, P. (2005). Tactical-decision learning model and students' practices. Physical Education and Sport Pedagogy, 10, 255-269.

Hadwin, A., Järvelä, S., \& Miller, M. (2018). Self-regulation, co-regulation, and shared regulation in collaborative learning environments. In D. H. Schunk \& J. A. Greene (Éds.), Educational psychology handbook series. Handbook of self-regulation of learning and performance (p. 83-106). New York, NY: Routledge/Taylor \& Francis Group.

Harvey, S. \& Jarrett, K. (2014). A review of the game-centered approaches to teaching and coaching literature since 2006. Physical Education and Sport Pedagogy, 19, 278300 .

Järvelä. S., Järvenoja, H., Malmberg, J., \& Hadwin, A. F. (2013). Exploring socially shared regulation in the context of collaboration. Journal of Cognitive Education and Psychology, 12, 267-286.

Johsua, S., \& Lahire, B. (1999). Pour une didactique sociologique. Éducation et Société, 4, 29-56.

Kawa, A. (1998). Le football en éducation physique au collège. Poitiers : CRDP de PoitouCharentes.

Kirk, D., \& McPhail, A. (2002). Teaching games for understanding and situated learning: Rethinking the Bunker-Thorpe model. Journal of Teaching in Physical Education, 21, 177-192.

Kozanitis, A. (2005). Les principaux courants théoriques de l'enseignement et de l'apprentissage : un point de vue historique. Montréal (QC, Canada) : Bureau 
d'Appui Pédagogique, École polytechnique de Montréal. Consulté sur : http://comeniusmathematiques.portail15.fr/wpcontent/uploads/2015/04/05 camprodon annexe5 Extrait historique approche enseignement.pdf

Mahlo, F. (1969). Acte tactique en jeu. Paris : Vigot.

Marle, P., \& Gréhaigne, J.-F. (2009). Football. Consulté sur www.contrepied.net

McBride, R. E. (1991). Critical thinking - An overview with implications for physical education. Journal of Teaching in Physical Education, 11, 112-125

Memmert, D., \& Roth, K. (2007). The effects of non-specific and specific concepts on tactical creativity in team ball sports. Journal of Sports Sciences, 25, 1423-1432.

Menaut, A. (1998). Le réel et le possible dans la pensée tactique. Talence: Presses Universitaires de Bordeaux.

Mérand, R. (1977). Considérations sur une problématique de rénovation des contenus de l'Éducation Physique en rapport avec les activités sportives contemporaines. In CPS de la FSGT (Éd.), L'éducateur face à la haute performance olympique (pp.0527). Paris : Sport et Plein air.

Mérand, R. (1984). Contribution à l'évaluation des connaissances et des capacités d'analyse des activités pratiquées. In SNEP (Éd.), L'évaluation en E.P.S (pp. 206207). Paris : SNEP.

Mouchet, A., Amans-Passaga, C., \& Gréhaigne, J.-F. (2010). L'approche technologique. In M. Musard, M. Loquet, \& G. Carlier (Éds.), Sciences de l'intervention en EPS et en sport. Résultats de recherches et fondements théoriques (pp. 201-222). Paris : Éditions EP\&S.

Nadeau, L., Gréhaigne, J.-F., \& Godbout, P. (2017). Developing tactical knowledge with the help of support players: An illustration in ice hockey. International Journal of Physical Education, 54(1), 22-33.

Nadeau, L., Richard, J.-F., \& Godbout, P. (2008). The validity and reliability of a performance assessment procedure in ice hockey. Physical Education and Sport Pedagogy, 13, 65-83.

Ochanine, D. (1978). Le rôle des images opératives dans la régulation des activités de travail. Psychologie et Éducation, 2, 63-72.

Pallascio, R. \& Lafortune, L. (Éds.). (2000). Pour une pensée réflexive en éducation. Québec : Presses de l'Université du Québec.

Perrenoud, P. (1994). Métier d'élève et sens du travail scolaire. Paris : ESF. 
Piaget, J. (1923). Le langage et la pensée chez l'enfant. Paris : Delachaux et Niestlé.

Piaget, J. (1969). Psychologie et pédagogie. Paris : Denoël.

Poggi, M.-P. (2016). Handball scolaire pour les filles en EPS. Quels risques d'inégalités ? In J.-F. Gréhaigne (Éd.), Les objets de la technique. De la compétence motrice à la tactique individuelle (pp. 271-286). Besançon : PUFC.

Poggi, M.-P., \& Moniotte J. (2011). Quelle place pour le sociologique dans les recherches en didactique de l'EPS ? Éducation et Didactique, 5(1), 29-44.

Ramos, A., Coutinho, P., Davids, K., \& Mesquita, I. (2020). Developing players' tactical knowledge using combined constraints-led and step-game approaches. A longitudinal action-research study. Research Quarterly for Exercise and Sport. Advance online publication.

Resnick, L. B. (1987). Education and learning to think. Washington: National Academy Press.

Richard, J.-F., Godbout, P., Tousignant, M., \& Gréhaigne, J.-F. (1999). The try-out of a team-sport assessment procedure in elementary and junior high school PE classes. Journal of Teaching in Physical Education, 18, 336-356.

Schwager, S., \& Labate, C. (1993). Teaching for critical thinking in physical education. Journal of Physical Education, Recreation and Dance, 64(5), 24-26.

Tan, C. (2017). Teaching critical thinking: Cultural challenges and strategies in Singapore. British Educational Research Journal, 43, 988-1002.

Tishman, S., \& Perkins, D. (1995). Critical thinking and physical education. Journal of Physical Education, Recreation and Dance, 66(6), 24-30.

Uribe Enciso, O. L., Uribe Enciso, D. S., \& Vargas-Daza, M. (2017). Critical thinking and its importance in education: Some reflections. Rastros Rostros, 19(34), 78-88.

Vidal-Gomel, C., \& Rogalski, J. (2007). La conceptualisation et la place des concepts pragmatiques dans l'activité professionnelle et le développement des compétences. @ctivités, 4(1), 49-84.

Vygotski, L. S. (1985). Pensée et langage. Paris : Éditions Sociales. (Travail original publié en 1934).

Wallian, N., \& Chang, C. W. (2007). Sémiotique de l'action motrice et des activités langagières : vers une épistémologie des savoirs co-construits en sports collectifs. In J.F. Gréhaigne (Éd.), Configurations du jeu, débat d'idées et apprentissage des sports collectifs (pp. 145-164). Besançon : Presses de l'Université de FrancheComté. 
Wallian, N., \& Gréhaigne, J.-F. (2004). Vers une approche sémio-constructiviste des apprentissages moteurs. In G. Carlier (Éd.), Si l'on parlait du plaisir d'enseigner l'éducation physique (pp. 167-179). Montpellier : AFRAPS.

Wallian, N., Poggi, M.-P., \& Chauvin-Vileno, A. (Éds.). (2008). Action, interaction, intervention. À la croisée du langage, de la pratique et des savoirs. Bern : Peter Lang.

Walliser, B. (1977). Systèmes et modèles. Introduction critique à l'analyse des systèmes. Paris : Le Seuil.

Zerai, Z. (2011). Apprentissage du handball chez les jeunes filles tunisiennes et françaises ; apport de la verbalisation. Thèse de doctorat non publiée en Sciences du Sport. Université de Franche-Comté, Besançon.

Zerai, Z., Gréhaigne, J.-F., \& Godbout, P. (2011). Des signes de ressemblance dans les configurations du jeu. In J.F. Gréhaigne (Éd.), Des signes au sens. Le jeu, les indices, les postures et les apprentissages dans les sports collectifs à l'école (pp. 73-84). Besançon : Presses de l'Université de Franche-Comté.

Zerai, Z., Gréhaigne, J.-F., \& Godbout, P. (2021). Débat, conceptualisation et éducation physique et sportive. eJRIEPS, 48, 29-47. 